\title{
Metachronous Carcinoma at the Colostomy Site After Abdominoperineal Resection of Rectal Cancer
}

\author{
Young Sun Choi ${ }^{1}$, Kil-young Lee ${ }^{2}$, Youn Young Park², Hyung Jin Kim ${ }^{1}$ Jaeim Lee \\ ${ }^{1}$ Department of Surgery, Eunpyeong St. Mary's Hospital, College of Medicine, The Catholic University of Korea, Seoul; ${ }^{2}$ Department of \\ Surgery, Uijeongbu St. Mary’s Hospital, College of Medicine, The Catholic University of Korea, Uijeongbu, Korea
}

Metachronous carcinoma at the colostomy site is very rare after abdominoperineal resection. A 53-year-old male patient underwent an abdominoperineal resection 6 years earlier for rectal cancer developed metachronous carcinoma at the site of stoma. A portion of the colon, including the stoma and the surrounding skin, was resected and a new stoma was created in the transverse colon. Although the occurrence of carcinoma at the stoma site is a rare condition, careful observation for the stoma and colonoscopy for surveillance are necessary.

Keywords: Rectal neoplasms; Adenocarcinoma; Second primary neoplasms; Surgical stomas; Colostomy

\section{INTRODUCTION}

Adenocarcinoma occurring at the site of a colostomy after abdominoperineal resection is very rare. The common metastatic sites after colorectal cancer resection are liver, peritoneum, lung, pelvis, and bone in decreasing frequency. The interval from initial rectal cancer surgery to metastasis is variable. Tumor metastasis occurs in various mechanisms including direct invasion, lymphatic and hematogenous spread. However, the exact mechanism of colostomy site metastasis is uncertain. We report a case of carcinoma that arose at the colostomy site 6 years after abdominoperineal resection. This study was approved by the Institutional Review Board of the Uijeongbu St. Mary's Hospital, College of Medicine, The Catholic University of Korea (No. UC13ZISE0112). Writitne informed consent was obtained for publication of this case report and accompanying images.

Received: Mar 16, 2020 - Revised: May 19, 2020 • Accepted: May 9, 2021 Correspondence to: Jaeim Lee, M.D.

Department of Surgery, Uijeongbu St. Mary's Hospital, College of Medicine, The Catholic University of Korea, 271 Cheonbo-ro, Uijeongbu 11765, Korea Tel: +82-31-820-5020, Fax: +82-31-847-2717

E-mail: lji96@catholic.ac.kr

ORCID: http://orcid.org/0000-0002-4074-3904

(C) 2021 The Korean Society of Coloproctology

This is an open-access article distributed under the terms of the Creative Commons Attribution NonCommercial License (https://creativecommons.org/licenses/by-nc/4.0) which permits unrestricted noncommercial use, distribution, and reproduction in any medium, provided the original work is properly cited.

\section{CASE REPORT}

A 53-year-old male patient visited the emergency department for the difficulty of defecation and stoma obstruction (Fig. 1). Six years ago, he was diagnosed with large bowel obstruction which results from a large rectal cancer and single liver metastasis. $\mathrm{He}$ got 2 cycles of preoperative chemoradiotherapy. He had undergone abdominoperineal resection with high inferior mesenteric artery (IMA) ligation and left lateral liver segmentectomy. Histopathology of the original specimen revealed a T3 tumor with clear margins, 4 regional lymph nodes were retrieved with no lymph node involvement and no lymphovascular invasion. The resected liver specimen revealed metastatic adenocarcinoma from the rectum. He had undergone FOLFOX (fluorouracil, leucovorin, and oxaliplatin) chemotherapy after surgery. Colonoscopy evaluation was performed at 1 year and 30 months after the initial operation, and the results were normal. Serum carcinoembryonic antigen level, computed tomography (CT) scan of the abdomen and pelvis, and whole-body bone scan were performed every 6 months until 3 years and 6 months after surgery. Initial CT scan of the abdomen and pelvis in the emergency department demonstrated a fungating mass at the stoma site. Positron emission tomographic CT scan of the whole body showed 18F-fluorodeoxyglucose hot uptake at the stoma site only (Fig. 2). There was no evidence of metastatic disease elsewhere. Excisional biopsy of the mass at the stoma site revealed adenocarcinoma from colonic origin. A completion colectomy and en bloc resection of colostomy were performed and the proximal colon from the stoma was resected with 


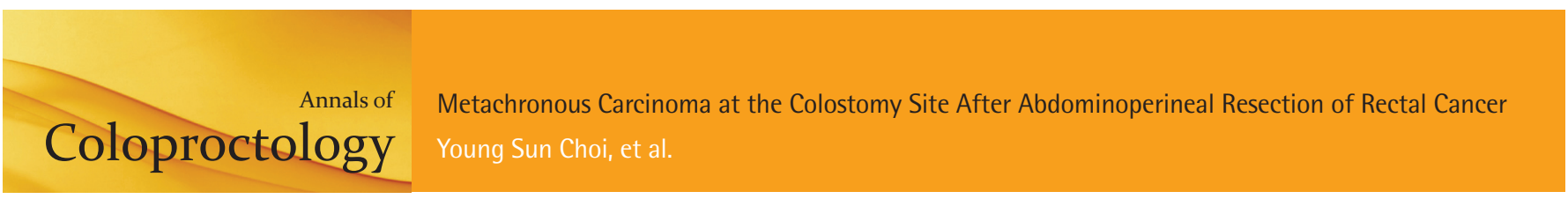

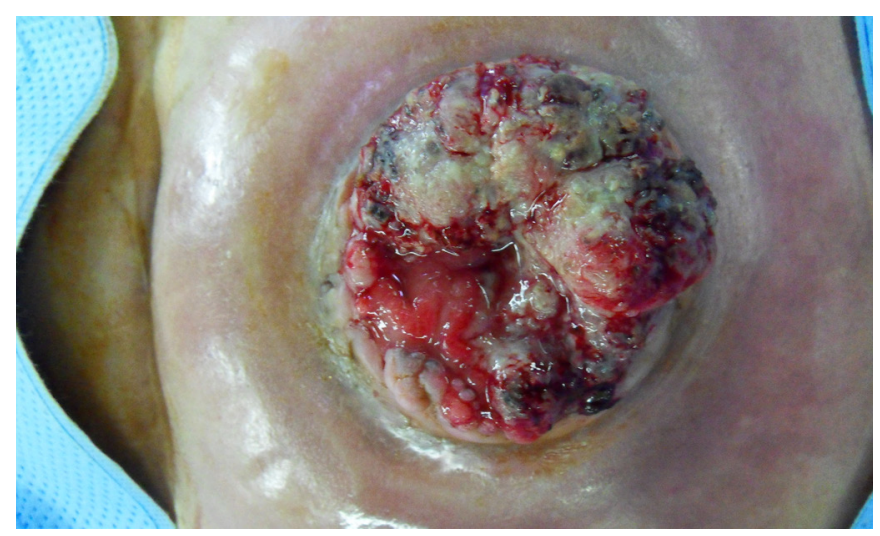

Fig. 1. Clinical photography showing a colostomy carcinoma with a resected specimen.

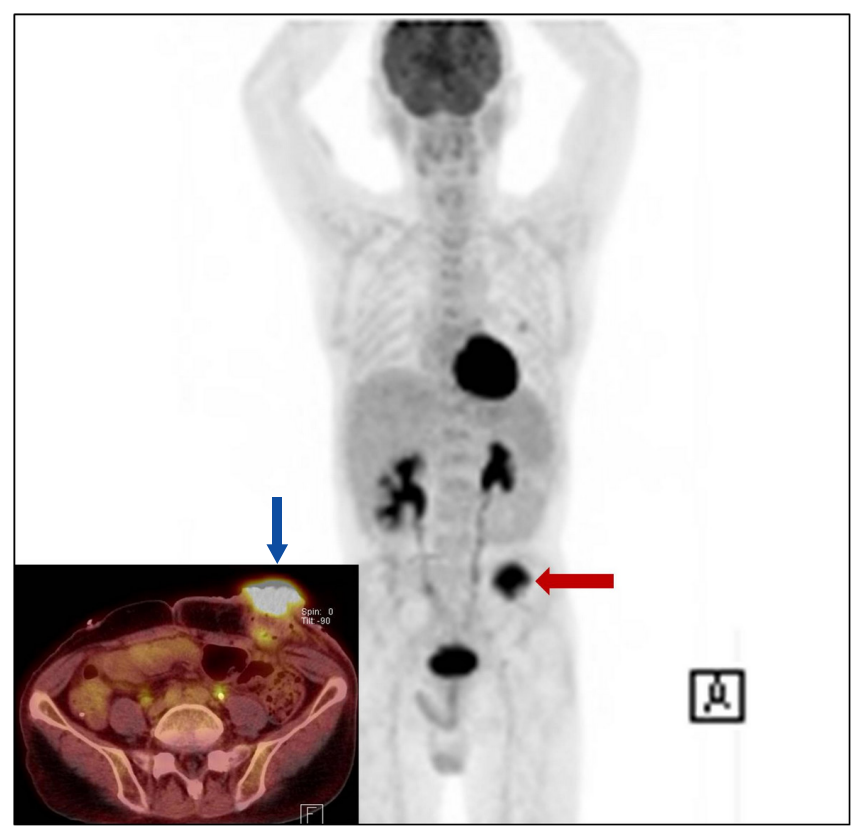

Fig. 2. The positron emission tomographic-computed tomography scan showed high levels of 18F-fluorodeoxyglucose uptake at the stoma site only (arrows).

a clear margin (Fig. 1). Transverse colostomy was made at the right upper quadrant. Histopathology of the specimen showed adenocarcinoma originating from the colon with a clear margin (Fig. 3). The carcinoma invaded into the proper muscle layer and had 5 lymph node involvement of total of 13 retrieved lymph nodes. He underwent adjuvant chemotherapy and 2 years later, the patient has no signs or symptoms of recurrence.

\section{DISCUSSION}

Metachronous colorectal cancer was defined as a primary colon

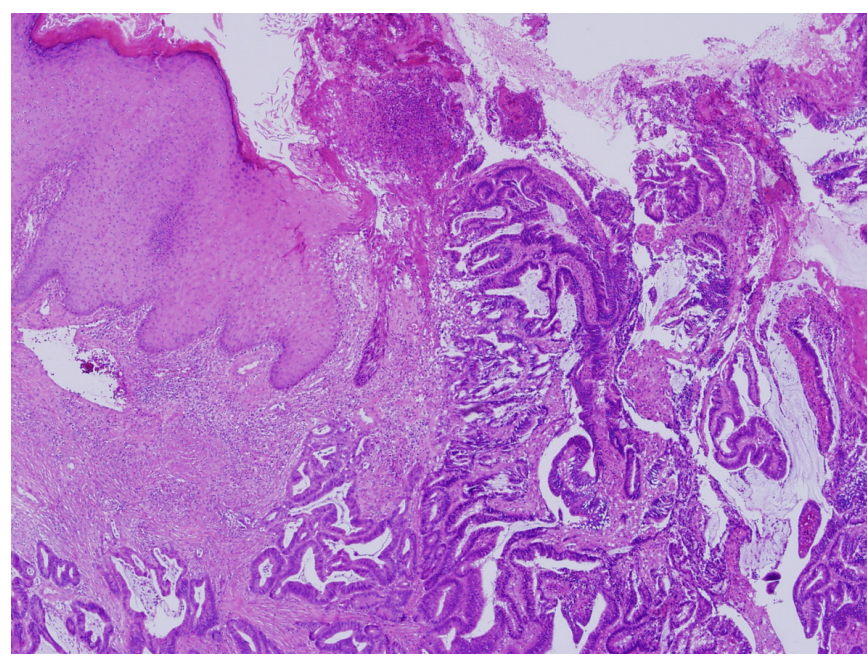

Fig. 3. The histopathology of the specimen showed complete excision of the stoma mass with clear margins and the microscopic examination showed an adenocarcinoma originating from the colon $(\mathrm{H} \& \mathrm{E}, \times 40)$.

cancer that occurs at any time after the surgical achievement of a clean colorectum [1]. Metachronous carcinoma at the stoma site is very rare after surgery $[2,3]$. About 12 cases in 11 case reports were found in the literature review. Most patients in reported cases undergo abdominoperineal resection and end colostomy. Although most colorectal metastases occur within 2 years from the primary tumor resection, the occurrence of metachronous carcinoma at the stoma site varies from 4 to 30 years after the initial resection of the primary tumor [2]. Clinical manifestation of carcinoma at the stoma site include tumor in 7 cases, bleeding and stoma stenosis in 2 cases each, and ulceration in 1 case [4].

Distant metastasis or recurrence of tumor generally results from hematogenous, lymphatic and direct spread of tumor cells. About the cause of the carcinoma occurrence at the colostomy site, various hypotheses were suggested. Takami et al. [5] suggested that the adenoma-carcinoma sequence on the basis of the presence of undetected, concomitant polyps. However, there was no evidence of adenoma at the stoma. Saegusa [6] suggested, because there was no sign of adenoma at the stoma that could be easily observed on a daily basis, the carcinoma at the stoma site may have been similar to the de novo metaplasia. Shibuya et al. [2] suggested a stoma stricture or ulceration was a cause of this occurrence. The stricture of the stoma may have been affected by bile acids and changes in enterobacteria that resulted from prolonged contact with stools. The stoma is also easily damaged than the intestinal mucosa because of exposure and unexpected compression. Vijayasekar et al. [7] suggested micro-metastasis left behind in lymph nodes along the IMA pedicle at the time of the abdominoperineal resection.

Literature has shown that the majority of recurrence from colorectal cancer is reported within 2 years after curative surgery 
[8]. A study by Galandiuk et al. [9] showed a median recurrence time of 16.7 months, with a range from 1 month to 7.5 years. Although the patient did not follow a routine surveillance program after surgery, studies performed during 3 years and 6 months after curative resection revealed normal including colonoscopy, serum carcinoembryonic antigen level, and CT scan of abdomen and pelvis. The initial abdominoperineal resection achieved R0 resection with high ligation of IMA and no lymph node involvement along the IMA was observed. It takes 5 to 10 years to develop adenocarcinoma from adenomas. Concurrent polyps are found in $21 \%$ to $55 \%$ of cases when colorectal cancer is diagnosed. Considering the results of surveillance study and the timing of occurrence of colostomy site carcinoma, there is a higher possibility of de novo cancer rather than recurrence of previous rectal cancer.

We performed not only en bloc resection of colostomy but also proximal colon resection and lymph node dissection, which found 5 positive lymph nodes. Although there is no consensus about standard treatment of metachronous carcinoma in colostomy site, if the tumor is highly suspected of de novo cancer, R0 resection including lymph node dissection should be considered. Additional lymph node resection can prevent poor oncologic outcome which may result from remnant microcarcinoma in lymph node. Adjuvant chemotherapy also needs to be considered according to histopathologic result.

Although the occurrence of carcinoma at the stoma site is a rare condition, it can occur in a stoma site. Most clinicians do not examine the stoma site until the patient's complaint. Careful inspection and recognition of the stoma are necessary.

\section{CONFLICT OF INTEREST}

No potential conflict of interest relevant to this article was reported.

\section{REFERENCES}

1. Fajobi O, Yiu CY, Sen-Gupta SB, Boulos PB. Metachronous colorectal cancers. Br J Surg 1998;85:897-901.

2. Shibuya T, Uchiyama K, Kokuma M, Shioya T, Watanabe Y, Moriyama $\mathrm{Y}$, et al. Metachronous adenocarcinoma occurring at a colostomy site after abdominoperineal resection for rectal carcinoma. J Gastroenterol 2002;37:387-90.

3. Chintamani, Singhal V, Bansal A, Bhatnagar D, Saxena S. Isolated colostomy site recurrence in rectal cancer-two cases with review of literature. World J Surg Oncol 2007;5:52.

4. Maeda C, Hidaka E, Shimada M, Shimada S, Nakahara K, Takayanagi $\mathrm{D}$, et al. Transverse colon cancer occurring at a colostomy site 35 years after colostomy: a case report. World J Surg Oncol 2015;13:171.

5. Takami M, Hanada M, Kimura M, Takeuchi N, Takada T. Adenocarcinoma arising at a colostomy site. Report of a case. Dis Colon Rectum 1983;26:50-2.

6. Saegusa K. A case of recurrent rectal carcinoma in the colostomy site. Chiba Med J 1986;62:122.

7. Vijayasekar C, Noormohamed S, Cheetham MJ. Late recurrence of large peri-stomal metastasis following abdomino-perineal resection of rectal cancer. World J Surg Oncol 2008;6:96.

8. Yaeger R, Cowell E, Chou JF, Gewirtz AN, Borsu L, Vakiani E, et al. RAS mutations affect pattern of metastatic spread and increase propensity for brain metastasis in colorectal cancer. Cancer 2015; 121:1195-203.

9. Galandiuk S, Wieand HS, Moertel CG, Cha SS, Fitzgibbons RJ Jr, Pemberton JH, et al. Patterns of recurrence after curative resection of carcinoma of the colon and rectum. Surg Gynecol Obstet 1992;174:27-32. 